\title{
ACCURATE RESONANT FREQUENCY COMPUTATION OF MULTISEGMENT RECTANGULAR DIELECTRIC RESONATOR ANTENNAS
}

\author{
Ç. S. Gürel
}

Department of Electrical and Electronics Engineering

Hacettepe University

Beytepe 06800, Ankara, Turkey

\section{H. Coşar}

Department of Electrical and Electronics Engineering

Computational Electromagnetics Research Center (BiLCEM)

Bilkent University

Bilkent 06800, Ankara, Turkey

\section{Ö. Akalın}

Department of Electrical and Electronics Engineering

Hacettepe University

Beytepe 06800, Ankara, Turkey

Abstract - In this study, multi-segment dielectric resonator antenna (MSDRA) is analyzed. A new formulation depending on the Weighted Average Model (WAM) is proposed for the determination of resonant frequency which is called as Modified Weighted Average Model (MWAM). According to the comparison of the results with experimental values and the results of the previous studies and simulation results it is shown that considerable improvement is obtained using this new formulation providing very small percentage errors for almost all cases.

\section{INTRODUCTION}

Various types of dielectric resonator antennas (DRAs) have been studied over past few years as they offer several advantages for wireless and mobile communication systems as high radiation efficiency, wide

Corresponding author: Ç. S. Gürel (cigdem@hacettepe.edu.tr). 
bandwidth, linear and circular polarization operations with the ease of fabrication, small size and low cost [1-11]. Especially the rectangular DRA (RDRA) configuration provides some advantages over other types. Mode degeneracy can be avoided by adjusting height/width/length ratio [12-14]. Additionally, the three dimensions of a rectangular DRA are used to control the bandwidth.

One of the most effective parameters of a DRA is its permittivity. As the permittivity gets lower, antenna bandwidth gets wider accompanied with reduced antenna-feedline coupling. This is a trade-off between bandwidth and coupling and to overcome this problem, multi-segment dielectric resonator antenna (MSDRA) was proposed [15-20]. MSDRAs have additional advantages such as smaller back lobe and more gain with respect to single segment DRAs.

In recent years, modeling of DRAs has been performed with different approaches. Some approximate models have generally been presented to predict the resonant frequency of such antennas. In the last years, two new methods have been introduced to find the resonant frequency of MSDRA. The first one is called as Modified Dielectric Waveguide Model (MDWM) which has been developed by Petosa et al. [15] and the second one is called as Weighted Average Model (WAM) developed by Rashidian et al. [16] similar to MDWM but in more complicated form. Average percentage error values while predicting resonant frequencies are generally high values around $4.43 \%$ and $2.5 \%$ for MDWM and WAM, respectively. Then, simpler formulation is presented for rectangular DRA in a very recent study which is valid only for single layered case [21].

In this paper, a new and simple method is proposed for the determination of resonant frequency of rectangular MSDRA. In this method, effective permittivity and effective dimensions are defined and used in a weighted resonant frequency formulation. This new method introduced for MSDRA analysis is called as Modified Weighted Average Model (MWAM) as the extension of WAM. By MWAM procedure, accurate resonant frequency values are obtained providing considerably small average percentage error values with respect to the MDWM and WAM which are around $1 \%$ or smaller. The overall performance of the study is also better than HFSS simulation results.

\section{THEORY}

Rectangular MSDRA configuration having three sections with different heights and permittivities is shown in Fig. 1.

The upper layer has low permittivity $\varepsilon_{u}$ and a height $h_{u}$. At the bottom, a microstrip feedline on a dielectric substrate having 


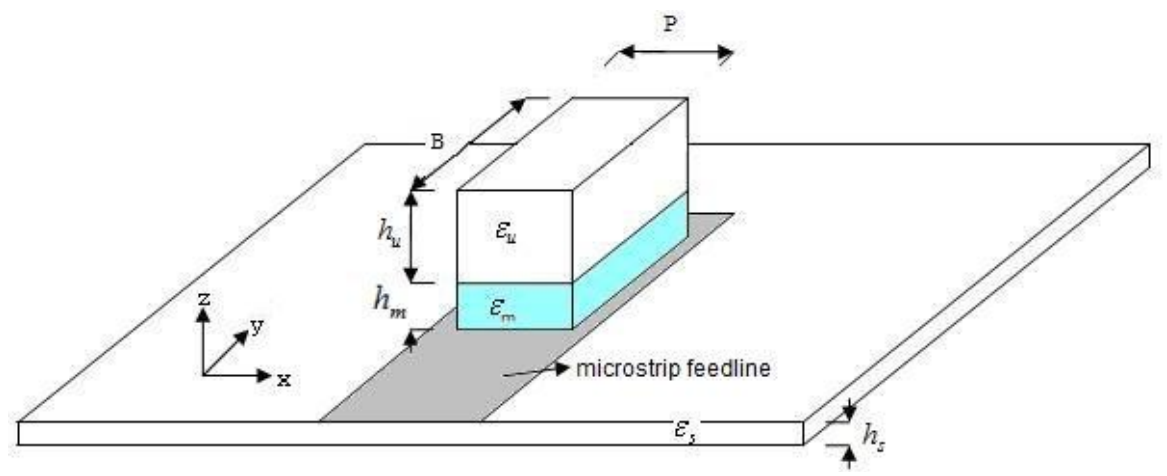

Figure 1. Multi-segment rectangular dielectric resonator antenna.

a low permittivity $\varepsilon_{s}$ and a height $h_{s}$ is used for coupling of the signals into the antenna. At the middle, one or more thin segments of different permittivity substrates are inserted to significantly improve the coupling performance. These inserts transform the impedance of the DRA to that of the microstrip feedline by concentrating the fields underneath the DRA. Although more than one insert can be added to obtain impedance match, only single insert with high permittivity $\varepsilon_{m}$ and height $h_{m}$ is used in order to reduce the complexity of the fabrication process and ultimately the cost. The antenna is working in its dominant mode, $T E_{111}$.

For a MSDRA in free space, to find the resonant frequency value for dominant mode, firstly effective permittivity is calculated as

$$
\varepsilon_{e f f}=\frac{\varepsilon_{s} h_{s}+\varepsilon_{m} h_{m}+\varepsilon_{u} h_{u}}{h_{s}+h_{m}+h_{u}}
$$

By using effective permittivity of MSDRA, effective dimensions are obtained as

$$
\begin{aligned}
& P_{\text {eff }}=P\left(1-\frac{1}{\varepsilon_{\text {eff }}}\right) \\
& B_{\text {eff }}=B\left(1-\frac{1}{\varepsilon_{\text {eff }}}\right)
\end{aligned}
$$

Then starting with DWM equations [14], characteristic equation for the mode wave number $k_{x}$ in $x$ direction is obtained as

$$
k_{x} \tan \left(\frac{k_{x} P_{\text {eff }}}{2}\right)=\sqrt{\left(\varepsilon_{x}-1\right) k_{0}^{2}-k_{x}^{2}}
$$

satisfying the relation

$$
k_{x}^{2}+k_{y}^{2}+k_{z}^{2}=\varepsilon_{x} k_{0}^{2}
$$


where $k_{x}, k_{y}$ and $k_{z}$ denote the wave numbers, respectively, at $x, y$ and $z$ directions. The $k_{y}$ and $k_{z}$ can be determined as

$$
\begin{aligned}
k_{y} & =\frac{\pi}{B_{\text {eff }}} \\
k_{Z} & =\left(\frac{\pi}{2\left(h_{s}+h_{m}+h_{u}\right)}\right)
\end{aligned}
$$

The analysed MSDRA structure consists of three different segments with permittivities $\varepsilon_{s}, \varepsilon_{m}$ and $\varepsilon_{u}$ as shown in Fig. 1. In (4) and (5), $\varepsilon_{s}, \varepsilon_{m}$ and $\varepsilon_{u}$ are used instead of $\varepsilon_{x}$ to calculate the weighted free space wavenumbers, $k_{0 s}, k_{0 m}, k_{0 u}$. After finding these wavenumbers, corresponding frequencies $f_{0 s}, f_{0 m}$, and $f_{0 u}$ can be found as

$$
f_{0 s, 0 m, 0 u}=\left(\frac{c}{2 \pi}\right) k_{0 s, 0 m, 0 u}
$$

According to the WAM, resonant frequency of MSDRA is calculated from

$$
f_{0}=\frac{h_{s} f_{0 s}+h_{m} f_{0 m}+h_{u} f_{0 u}}{h_{s}+h_{m}+h_{u}}
$$

In this study, instead of $(9), f_{o}$ is taken in the form

$$
f_{0}=\left\{\begin{array}{ll}
f_{c}\left(1+\frac{\varepsilon_{\text {eff }}}{500}\right) & 10<\varepsilon_{\text {eff }}<22 \\
f_{c}\left(1-\frac{\varepsilon_{\text {eff }}}{500}\right) & \text { elsewhere }
\end{array}\right\}
$$

where the frequency correction term $f_{c}$ is obtained as

$$
f_{c}=\left(\frac{h_{s} \sqrt[3 / 2]{f_{0 s}}+h_{m} \sqrt[3 / 2]{f_{0 m}}+h_{u} \sqrt[3 / 2]{f_{0 u}}}{h_{s}+h_{m}+h_{u}}\right)^{3 / 2}
$$

In (8), two different correction terms are proposed to improve the accuracy of the calculations according to the experimental results. Especially for the second case the results of the previous theories show considerable shift from the experimental results which generally correspond to high layer permittivities or thin layer thicknesses with low permittivities.

In the case of more layers than two, similar terms can be added to the (1), (7) and (11) for each new segment of the antenna. This modified formulation is called as "Modified Weighted Average Model (MWAM)". The resonant frequency results of MWAM, WAM and MDWM in addition to the HFSS simulation results are given in the next section for several rectangular MSDRA structures. 


\section{RESULTS}

In the MSDRA, the permittivity and the thickness of the insert layer affect the resonant frequency value. This effect was determined for nine different insert cases in [15].

In order to compare the present method MWAM and two previous methods MDWM [15] and WAM [17] and HFSS simulation results in terms of their accuracy, resonant frequency results of each case for an antenna having the parameters $P=2 \mathrm{~mm}, B=7.875 \mathrm{~mm}$, $h_{u}=3.175 \mathrm{~mm}, h_{S}=0.762 \mathrm{~mm}, \varepsilon_{u}=10$ and $\varepsilon_{s}=3$ are shown in Table 1 including experimental results. Corresponding percentage error values for each case are also included in this table.

It is seen from Table 1 that MDWM is only accurate when $h_{m}<$ $0.5 \mathrm{~mm}$. Generally, as $h_{m}$ is increased, amount of error in resonant frequency increases for MDWM. It is observed that WAM exhibits more accurate results than MDWM especially for high permittivity antennas. In row 4 of the Table 1 , it is observed that the results of MDWM and WAM are better than MWAM. This may be due to special case of thin layer thickness with intermediate layer permittivity value. It must be noted that for this case, percentage error value of HFSS simulation result is higher than the current method.

Table 1. Comparison of experimental and theoretical resonant frequency results of multi-segment rectangular DRA in GHz. $P=$ $2 \mathrm{~mm}, B=7.875 \mathrm{~mm}, h_{u}=3.175 \mathrm{~mm}, h_{s}=0.762 \mathrm{~mm}, \varepsilon_{u}=10$ and $\varepsilon_{s}=3$.

\begin{tabular}{|r|c|c|c|r|r|r|r|r|r|r|r|}
\hline DRA & $\begin{array}{c}h_{m} \\
(\mathrm{~mm})\end{array}$ & $\varepsilon_{m}$ & $\begin{array}{r}f_{0, \text { meas }} \\
{[15]}\end{array}$ & $\begin{array}{r}f_{0, M D W M} \\
{[15]}\end{array}$ & $\begin{array}{r}f_{0, W A M} \\
{[17]}\end{array}$ & $\begin{array}{r}f_{0, H F S S} \\
{[18]}\end{array}$ & $f_{0, M W A M}$ & $\begin{array}{r}\text { \%Error } \\
\text { MDWM }\end{array}$ & $\begin{array}{r}\text { \%Error } \\
\text { WAM }\end{array}$ & $\begin{array}{r}\text { \%Error } \\
\text { HFSS }\end{array}$ & $\begin{array}{r}\text { \%Error } \\
\text { MWAM }\end{array}$ \\
\hline 1 & 0.25 & 20 & 14.7 & 14.5 & 15.2 & 14.9 & 14.8 & 1.4 & -3.4 & -1.4 & -0.7 \\
\hline 2 & 0.635 & 20 & 14.5 & 14.0 & 14.3 & 14.7 & 14.4 & 3.4 & 1.4 & 1.4 & 0.7 \\
\hline 3 & 1 & 20 & 13.9 & 13.5 & 13.6 & 13.8 & 13.7 & 2.9 & 2.2 & 0.7 & 1.4 \\
\hline 4 & 0.25 & 40 & 14.7 & 14.5 & 14.9 & 15.4 & 15.0 & 1.4 & -1.4 & -4.8 & -2.0 \\
\hline 5 & 0.635 & 40 & 13.7 & 13.9 & 13.7 & 13.6 & 13.8 & -1.5 & 0.0 & -0.7 & -0.7 \\
\hline 6 & 1 & 40 & 12.9 & 13.2 & 12.8 & 12.8 & 12.9 & -2.3 & 0.8 & -0.8 & 0.0 \\
\hline 7 & 0.25 & 100 & 14.7 & 14.5 & 14.5 & 14.9 & 14.6 & 1.4 & 1.4 & 1.4 & 0.7 \\
\hline 8 & 0.635 & 100 & 13.1 & 13.8 & 13.0 & 13.0 & 13.2 & -5.3 & 0.8 & -0.8 & -0.8 \\
\hline 9 & 1 & 100 & 10.8 & 13.0 & 12.0 & 10.9 & 11.0 & -21.0 & -11.1 & 0.9 & -1.9 \\
\hline
\end{tabular}


In Table 2, total absolute percentage error values and resulting average percentage error are given for all methods. It is clearly shown that the average percentage error value of the current method is better than the HFSS simulation results depending on the simple formulations.

It is observed that the results of the current method, MWAM, resonant frequencies are very close to measured values with respect to other methods. MWAM has considerable improvements in almost all cases over WAM. Average percentage error values of these methods are evaluated as $4.43 \%, 2.5 \%, 1.42 \%$ and $0.99 \%$ for MDWM, WAM, HFSS and MWAM, respectively.

In Fig. 2, resonant frequency shift of RDRA with the inclusion of the new insert layer having permittivity $\varepsilon_{k}$ and thickness $h_{k}$ on top

Table 2. Comparison of total absolute and percentage error values of previous theories and MWAM.

\begin{tabular}{|c|c|c|c|c|}
\hline & $\begin{array}{c}\text { MDWM } \\
{[15]}\end{array}$ & $\begin{array}{c}\text { WAM } \\
{[17]}\end{array}$ & $\begin{array}{c}\text { HFSS } \\
{[18]}\end{array}$ & MWAM \\
\hline Total Absolute P. E. & 39.9 & 22.30 & 12.78 & 8.90 \\
\hline Average P. E. & 4.43 & 2.50 & 1.42 & 0.99 \\
\hline
\end{tabular}

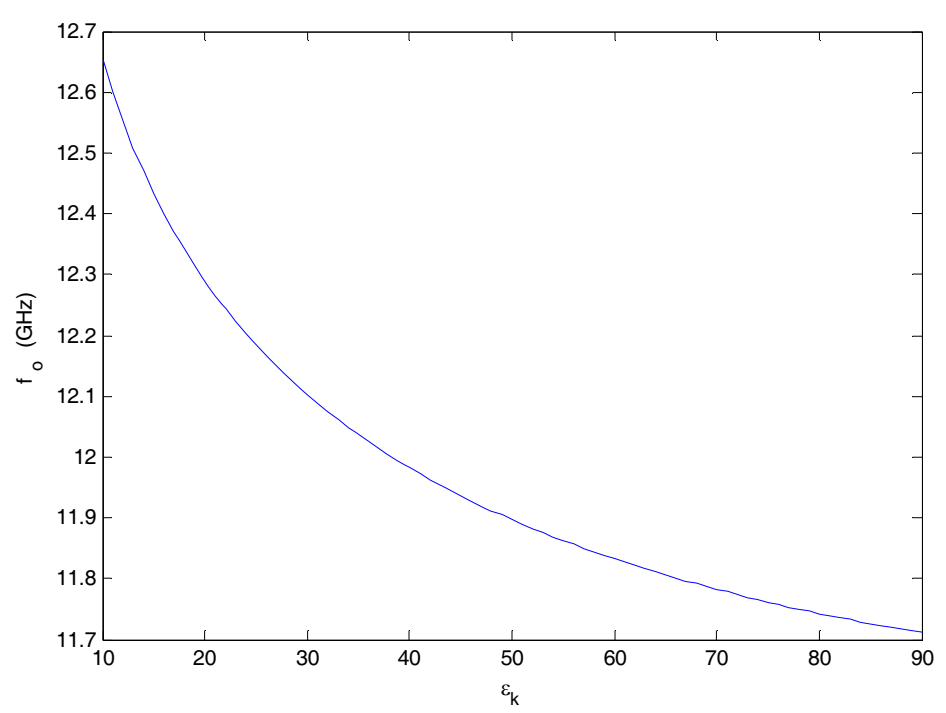

Figure 2. Resonant frequency shift with additional insert layer permittivity $\varepsilon_{k} \cdot P=2 \mathrm{~mm}, B=7.875 \mathrm{~mm}, h_{u}=3.175 \mathrm{~mm}, h_{s}=$ $0.762 \mathrm{~mm}, h_{m}=1 \mathrm{~mm}, h_{k}=0.5 \mathrm{~mm}, \varepsilon_{u}=10$ and $\varepsilon_{s}=3, \varepsilon_{m}=40$. 
of the first insert layer having permittivity $\varepsilon_{m}$ and thickness $h_{m}$ is shown for different insert layer permittivities. It is observed in the figure that resonant frequency of four layered antenna decreases as the insert permittivity increases. For this figure structural parameters of the RDRA is chosen to satisfy the $10<\varepsilon_{\text {eff }}<22$ range which is the mostly used range in practical applications. The effective permittivity values corresponding to rows $2-8$ of Table 1 are also obtained in this range.

It must be noted that for other effective permittivity values near to critical values such as $\varepsilon_{\text {eff }} \approx 10,22$, average of two results obtained with two different correction terms in (10) can be used in resonant frequency calculations to improve accuracy of the results.

\section{CONCLUSION}

In this study, an accurate method is developed to calculate the resonant frequency of multi-segment rectangular DRA by using effective structural parameters and weighted resonant frequency formulation. Considerable improvements on previous methods and HFSS simulation results are obtained using simple formulas within very small computational time. The formulas presented in this study can also be used in the calculations of other antenna parameters, and this method can be generalised to similar DRA configurations.

\section{REFERENCES}

1. Petosa, A., A. Ittipiboon, Y. M. M. Antar, and D. Roscoe, "Recent advances in dielectric resonator antenna technology," IEEE Antennas and Propagat Magazine, Vol. 40, No. 3, 35-48, Jun. 1998.

2. Luk, K. M. and K. W. Leung, Dielectric Resonator Antennas, Research Studies Press LTD., London, 2003.

3. Rezaei, P., M. Hakkak, and K. Forooraghi, "Dielectric resonator antenna for wireless LAN applications," IEEE Antennas and Propagation Society International Symposium, Vol. 2, 1005-1008, Jul. 2006.

4. Coulibaly, Y., T. A. Denidni, and L. Talbi, "Design of a broadband hybrid dielectric resonator antenna for X-band applications," Journal of Electromagnetic Waves and Applications, Vol. 20, No. 12, 1629-1642, 2006.

5. Saed, M. A. and R. Yadla, "Microstrip-fed low profile and compact 
dielectric resonator antennas," Progress In Electromagnetics Research, PIER 56, 151-162, 2006.

6. Kumar, A. V. P., V. Hamsakutty, J. Yohannan, and K. T. Mathew, "Microstripline FED cylindrical dielectric resonator antenna with a coplanar parasitic strip," Progress In Electromagnetics Research, PIER 60, 143-152, 2006.

7. Abdulla, P. and A. Chakrabarty, "Rectangular waveguidefed hemispherical dielectric resonator antenna," Progress In Electromagnetics Research, PIER 83, 225-244, 2008.

8. Zainud-Deen, S. H., H. A. El-Azem Malhat, and K. H. Awadalla, "A single-feed cylindrical superquadratic dielectric resonator antenna for circular polarization," Progress In Electromagnetics Research, PIER 85, 409-424, 2008.

9. Ding, Z., S.-Q. Xiao, Y. Bai, and B.-Z. Wang, "Hemisphere dielectric resonator pattern reconfigurable antenna and its linear phased array," Progress In Electromagnetics Research Letters, Vol. 6, 183-192, 2009.

10. Sreekantan, S., Y. K. Ling, Z. A. Ahmad, M. F. Ain, M. A. Othman, and S. I. S. Hassan, "Simulation and experimental investigations on rectangular, circular and cylindrical dielectric resonator antenna," Progress In Electromagnetics Research C, Vol. 7, 151-166, 2009.

11. Fayad, H. and P. Record, "Mutual coupling between a pair of identical beam steering dielectric resonator antennas," Progress In Electromagnetics Research B, Vol. 14, 23-44, 2009.

12. Mongia, R. K., A. Ittipiboon, and M. Cuhaci, "Measurement of radiation efficiency of dielectric resonator antennas," IEEE Microwave Guided Wave Lett., Vol. 4, 80-82, Mar. 1994.

13. Mongia, R. K. and P. Bhartia, "Dielectric resonator antennas: Review and general design relations for resonant frequency and bandwidth," Int. J. Microw. Millim. Wave Comp. Aided Eng., Vol. 4, 230-247, 1994.

14. Mongia, R. K. and A. Ittipiboon, "Theoretical and experimental investigations on rectangular dielectric resonator antennas," IEEE Trans. Antennas Propagat., Vol. 45, 1348-1356, Sept. 1997.

15. Petosa, A., N. Simons, R. Siushansian, A. Ittipiboon, and M. Cuhaci, "Design and analysis of multisegment dielectric resonator antennas," IEEE Trans. Antennas Propagat., Vol. 48, 738-742, 2000.

16. Rashidian, A., K. Forooraghi, and M. R. Tayfeh, "A new method for calculating the resonant frequency of multi-segment 
dielectric resonator antennas," 13th IEEE Int. Conf. Microwave Telecommun. Tech., 397-399, Crimea, Ukraine, 2003.

17. Rashidian, A., K. Forooraghi, and M. R. Tayfeh, "Design algorithm of multi-segment dielectric resonator antennas," 4th IEEE Int. Conf. Microw. Millim. Wave Tech., 134-137, 2004.

18. Rashidian, A., K. Forooraghi, and M. T. Aligodarz, "Investigations on two-segment dielectric resonator antennas," Microw. Opt. Tech. Lett., Vol. 45, 533-537, Jun. 2005.

19. Ittipiboon, A., D. Roscoe, A. Petosa, R. K. Mongia, M. Cuhaci, and R. Larose, "Broadband nonhomogeneous multisegmented dielectric resonator antenna system," U.S. Patent No. 5952972.

20. Rezaei, P., M. Hakkak, and K. Forooraghi, "Design of wideband dielectric resonator antenna with a two-segment structure," Progress In Electromagnetics Research, PIER 66, 111-124, 2006.

21. Gurel, C. S. and H. Cosar, "Efficient method for resonant frequency computation of rectangular dielectric resonator antennas," Microw. Opt. Tech. Lett., Vol. 51, 1706-1708, Jul. 2009. 